\title{
Modeling the Effect of Antipsychotic Drugs on Body Weight of Psychiatric Patients
}

\author{
Soyinka A. Taiwo ${ }^{1 *}$ and Oladunni O.F. Ololade ${ }^{2^{* *}}$ \\ ${ }^{1}$ Department of Statistics, Federal University of Agriculture Abeokuta, Ogun \\ state Nigeria. \\ ${ }^{2}$ Department of Nutrition, Federal Neuropsychiatric Hospital Aro Abeokuta, \\ Ogun state Nigeria. \\ *Corresponding Author: soyinkataiwo@yahoo.ie
}

Received: $28^{\text {th }}$ June 2018/ Revised: 30 ${ }^{\text {th }}$ Sep. 2018 / Published: 30 ${ }^{\text {th }}$ Dec. 2018 CIAppStat-SL2018

\begin{abstract}
Various studies have confirmed the significance increase in weight between baselines and follow up after commencement of antipsychotic drugs. Both Atypical Antipsychotic Drugs (AAD) and Typical Antipsychotic Drugs (TAD) have shown the tendency of inducing increase in the fat content of the body. However the modeling of the exact size of the weight increase beyond probable interval estimate is a necessity for clearer understanding; that will foster easy control of weight gain in psychiatric patient. This study thus established the claim from previous studies and presents the odds of $A A D$ inducing weight gain relative to TAD. The study used a composite trinomial and bernoulli distribution to describe the interest variables (IV's) body mass index (BMI) and the drug type. The composite function parameters and its standard errors were obtained using maximum likelihood estimation (MLE) method and fisher approach respectively. The study further used the continuation ratio model to determine the exact weight gain proportion due to $A A D$ relative to TAD. The study was carried out at the Out-patient clinic of the Federal Neuropsychiatric Hospital Aro, Abeokuta Nigeria, within a period of six weeks between baseline and follow up. A total of two hundred and seventy patients participated in the study after their consent and willingness were obtained. The difficulty to control the food intake of the patient at home is a limitation to the study. Patients that have had reasons to switch were excluded from the study. A graphical representation was used to explain the exact behavioural pattern of the weight gain and its relative effect due to AAD and TAD drugs. The study thus recommends a thorough follow up of healthy eating of food rich in fibers, fruits and vegetables along with indoor or outdoor physical exercises for psychiatric patients mostly on AAD to prevent future cardiovascular related diseases.
\end{abstract}

Keywords: Antipsychotic drugs, Body mass index, trinomial and bernoulli distribution, continuation ratio, odds function, nutritional control. 


\section{Introduction}

Antipsychotic medication (or neuroleptic) is a psychiatric medication primarily used to manage psychosis, particularly in schizophrenia and bipolar disorder. These medications should have minimum side effects profile for it to have high efficacy. There are two types of antipsychotic medication which are older or first-generation antipsychotic medications which are also referred to as conventional "typical" antipsychotics or "neuroleptics" and second-generation medications called "atypical" antipsychotics (Second-Generation Antidepressants, 2007). According to a research reviewed by the Agency for Healthcare Research and Quality (2013), both typical and atypical antipsychotics are used to treat symptoms of schizophrenia and the manic phase of bipolar disorder. Many "atypical" antipsychotic drugs (AADs) have been developed recently, which possess advantages over "typical" antipsychotic in terms of reduced adverse effects and efficacy (Second-Generation Antidepressants, 2007). These second-generation antipsychotics have gathered much literature about their profound effects on weight gain and the development of metabolic disorders. Bernstein (1987) noted that weight gain is one of the major side effects of many antipsychotic drugs. Further, Green et al. (2000) observed that AADs induced weight gain is associated with increased morbidity, mortality and reduced quality of life while this weight gain may interfere with compliance of the drugs, which may predispose patient to relapse (Agboola et al, 2018). Therefore, weight gain has thus emerged as a major side effect of AADs (Baptista et al. 2002b, 2004a; Goudieet et al. 2005). In addition to the weight gain induced by these AADs, it has been reported that those patients may suffer from metabolic abnormalities including hyperinsulinemia, hyperglycemia and diabetes (Henderson 2002; Holt et al. 2004). The physical health of patients with Mental illness is an important issue to consider when preparing a comprehensive treatment (Mas-Expósito et al, 2012; Chukwujekwu and Olose, 2019; Lopez-Munor et al, 2019). Thus, obesity is a threat to health and longevity (Allison et al, 1995). Hence, this study considered the graphical behaviour of weight gain due to AADs and TADs along with its odds to suggest a proper nutritional control of weight gain and thereby controlling the effect of induced weight gain by the antipsychotic drugs. This will prevent patients suffering from cardiovascular related illnesses. A total of two hundred and seventy patients participated in the study after their consent and willingness were obtained. There BMI were obtained at recruitment visit. During follow up visits, the BMIs were again recorded and grouped the patients appropriately. Some have their drugs changed during the course of the research due to some unbearable side effect(s) and/or lack of improvement and those ones were excluded from the study. Likewise, those that did not show up for follow up, were also excluded. 
Recruitment BMI as well as BMIs of two follow up visits were recorded and its average obtained for the final data analysis.

\section{Mathematical relation}

Let random variables $x \in X, y \in Y$ and $z \in Z$ denotes BMI groups underweight $(x)<18$, normal $(y) \geq 18-<25$ and overweight $(z) \geq 25$ while s and $\mathrm{t}$ denotes the drug types AAD and TAD respectively; owing that each patient are restricted to take one and only one of the two antipsychotic drugs, then the composite functional representation from a trinomial-Bernoulli (Mood, 1974) probability mass function can be represented as

$p_{\text {rob }}(x, y, z)=\frac{\left(18+z_{k}-x_{i}-y_{j}\right) !}{\left(18-x_{i}\right) !\left(25-y_{j}\right) !\left(z_{k}-25\right) !}\left\{p_{1}^{s}\left(1-p_{1}\right)^{t}\right\}^{18-x}\left\{p_{2}^{s}\left(1-p_{2}\right)^{t}\right\}^{25-y}\left\{p_{3}^{s}\left(1-p_{3}\right)^{t}\right\}^{z-25}$

where $p_{1}, p_{2}, p_{3}$ are the parameters with constraints $p_{1}{ }^{s}\left[1-p_{1}\right]^{t}+p_{2}{ }^{s}\left[1-p_{2}\right]^{t}+p_{3}{ }^{s}\left[1-p_{3}\right]^{t}=1$.

For $r=r_{1}+r_{2}+r_{3}$ several patients with diverse BMIs ranging from $x_{1}, x_{2}, . ., x_{r_{1}}$ for underweight $(x), y_{1}, y_{2}, . ., y_{r_{2}}$ for normal $(y)$ and $z_{1}, z_{2}, . ., z_{r_{3}}$ for overweight $(z)$. Then the likelihood function from (1) can be obtained as

$$
\begin{aligned}
& L\left(x, y, z \mid p_{1}, p_{2}, p_{3}\right)=\prod_{i=1}^{r_{1}} \prod_{j=1}^{r_{2}} \prod_{k=1}^{r_{3}}\left\langle\frac{\left(18+z_{k}-x_{i}-y_{j}\right)_{-} !}{\left(18-x_{i}\right) !\left(25-y_{j}\right) !\left(z_{k}-25\right) !}\right\rangle \\
& \left\{p_{1}^{s}\left(1-p_{1}\right)^{t}\right\}^{18 r_{1}-\sum_{i=1}^{r_{1}} x_{i}}\left\{p_{2}^{s}\left(1-p_{2}\right)^{t}\right\}^{25 r_{2}-\sum_{j=1}^{r_{2}} y_{j}}\left\{p_{3}^{s}\left(1-p_{3}\right)^{t}\right\}^{r_{3}} z_{k}-25 r_{3}
\end{aligned}
$$

Note for simplicity, it will be assumed that the patients were treated with only AAD $s=1, t=0$ and TAD $s=0, t=1$ intermittently. Obtaining the maximum likelihood value of the parameter $p_{1}, p_{2}, p_{3}$ by simultaneous solution of

$$
\begin{aligned}
& \frac{d L\left(x, y, z \mid p_{1}, p_{2}, p_{3}\right)}{d p_{1}}=0 \\
& \frac{d L\left(x, y, z \mid p_{1}, p_{2}, p_{3}\right)}{d p_{2}}=0 \quad \text { and } \\
& \frac{d L\left(x, y, z \mid p_{1}, p_{2}, p_{3}\right)}{d p_{3}}=0
\end{aligned}
$$

then 


$$
\frac{\partial L\left(x, y, z \mid p_{1}, p_{2}, p_{3}\right)}{\partial p_{1}}=\frac{\sum_{i=1}^{r_{1}}\left(18-x_{i}\right)}{p_{1}}-\frac{\sum_{k=1}^{r_{3}}\left(z_{k}-25\right)}{1-p_{1}-p_{2}}
$$

Which implies

$$
\frac{1-p_{1}-p_{2}}{p_{1}}=\frac{\sum_{k=1}^{r_{3}}\left(z_{k}-25\right)}{\sum_{i=1}^{r_{1}}\left(18-x_{i}\right)} \text {. }
$$

Similarly, $\frac{1-p_{1}-p_{2}}{p_{2}}=\frac{\sum_{k=1}^{r_{3}}\left(z_{k}-25\right)}{\sum_{i=1}^{r_{2}}\left(25-y_{j}\right)}$

can be given as

$$
\frac{p_{1}}{p_{2}}=\frac{\sum_{i=1}^{r_{1}}\left(18-x_{i}\right)}{\sum_{i=1}^{r_{2}}\left(25-y_{j}\right)} .
$$

However, using fisher information approach and obtaining the variance which has the reciprocal of the Cramer Rao Lower Bound

$$
\begin{aligned}
& \operatorname{Var}\left(p_{1}\right)=\left(-E \frac{\partial^{2} L\left(x, y, z \mid p_{1}, p_{2}, p_{3}\right)}{\partial p_{1}^{2}}\right)^{-1}=\left(\frac{r_{1}}{p_{1}}+\frac{r_{3}}{1-p_{1}-p_{2}}\right)^{-1}, \\
& \operatorname{Var}\left(p_{2}\right)=\left(\frac{r_{2}}{p_{2}}+\frac{r_{3}}{1-p_{1}-p_{2}}\right)^{-1} \text { And } \\
& \operatorname{Var}\left(1-p_{1}-p_{2}\right)=\frac{1-p_{1}-p_{2}}{r_{3}}
\end{aligned}
$$

The contingency table below is the categorical relationship between psychiatric diseases (Schizophrenia, Bipolar and Anxiety), BMI (underweight, normal and overweight) and drug type (AAD and TAD) where the number of patients in each interwoven category is the outcome. 
Table 1. Body Mass Index of Psychiatric patients on AAD and TAD

\begin{tabular}{|c|c|c|c|c|}
\hline \multirow{2}{*}{$\begin{array}{l}\text { Psychiatric } \\
\text { Diagnosis }\end{array}$} & \multirow{2}{*}{$\begin{array}{c}\text { Body } \\
\text { Mass Index }\end{array}$} & \multirow{2}{*}{$\begin{array}{l}\text { Summary } \\
\text { Statistics }\end{array}$} & \multicolumn{2}{|c|}{ Types of Drug Used } \\
\hline & & & AAD & TAD \\
\hline \multirow[t]{9}{*}{ Schizophrenia } & $\begin{array}{l}\text { Underweight } \\
(<18)\end{array}$ & Mean & 16.8108 & 16.8536 \\
\hline & & Frequency $(n)$ & 13 & 11 \\
\hline & & Sum & 218.54 & 185.39 \\
\hline & $\begin{array}{l}\text { Normal }(\geq 18 \\
-<25)\end{array}$ & Mean & 22.0356 & 19.7145 \\
\hline & & Frequency (n) & 9 & 40 \\
\hline & & Sum & 198.32 & 788.58 \\
\hline & $\begin{array}{l}\text { Overweight } \\
(\geq 25)\end{array}$ & Mean & 29.9619 & 28.6850 \\
\hline & & Frequency & 16 & 10 \\
\hline & & Sum & 479.39 & 286.85 \\
\hline \multirow[t]{9}{*}{ Bipolar } & $\begin{array}{l}\text { Underweight } \\
(<18)\end{array}$ & Mean & & 17.9220 \\
\hline & & Frequency (n) & & 5 \\
\hline & & Sum & & 89.61 \\
\hline & $\begin{array}{l}\text { Normal }(\geq 18 \\
-<25)\end{array}$ & Mean & 22.2255 & 21.1480 \\
\hline & & Frequency (n) & 29 & 10 \\
\hline & & Sum & 644.54 & 211.48 \\
\hline & $\begin{array}{l}\text { Overweight } \\
((\geq 25)\end{array}$ & Mean & 28.5388 & 29.4191 \\
\hline & & Frequency (n) & 8 & 11 \\
\hline & & Sum & 228.31 & 323.61 \\
\hline \multirow[t]{9}{*}{ Anxiety } & $\begin{array}{l}\text { Underweight } \\
(<18)\end{array}$ & Mean & 17.6814 & 17.97 \\
\hline & & Frequency (n) & 7 & 1 \\
\hline & & Sum & 123.77 & 17.97 \\
\hline & $\begin{array}{l}\text { Normal }(\geq 18 \\
-<25)\end{array}$ & Mean & 21.7844 & \\
\hline & & Frequency $(\mathrm{n})$ & 9 & \\
\hline & & Sum & 196.06 & \\
\hline & $\begin{array}{l}\text { Overweight } \\
((\geq 25)\end{array}$ & Mean & 28.6443 & 31.6664 \\
\hline & & Frequency $(\mathrm{n})$ & 7 & 11 \\
\hline & & Sum & 200.51 & 348.33 \\
\hline
\end{tabular}




\begin{tabular}{|l|l|c|c|c|}
\hline Total & $\begin{array}{l}\text { Underweight } \\
(<18)\end{array}$ & Mean & 17.12 & 17.23 \\
\cline { 2 - 5 } & & Frequency (n) & 20 & 17 \\
\cline { 2 - 5 } & $\begin{array}{l}\text { Normal }(\geq 18 \\
-<25)\end{array}$ & Sum & 342.31 & 292.97 \\
\cline { 2 - 5 } & & Frequency (n) & 47 & 20.00 \\
\cline { 2 - 5 } & & Sum & 1038.92 & 1000.06 \\
\cline { 2 - 5 } & $\begin{array}{l}\text { Overweight } \\
((\geq 25)\end{array}$ & Mean & 29.30 & 29.96 \\
\cline { 2 - 5 } & & Frequency (n) & 31 & 32 \\
\cline { 2 - 5 } & & Sum & 908.21 & 958.79 \\
\hline
\end{tabular}

Agboola et al, 2018; Agboola et al, 2020.

\section{Results and Discussion}

\section{Data Analysis}

(i) Atypical Antipsychotic Drugs (AAD)

$$
\begin{aligned}
& \frac{1-p_{1}-p_{2}}{p_{1}}=7.530243 \quad, \quad \frac{1-p_{1}-p_{2}}{p_{2}}=0.978909 \\
& \frac{p_{1}}{p_{2}}=0.129997
\end{aligned}
$$

Imposing the constraints, we have

$\left(p_{1}: p_{2}: 1-p_{1}-p_{2}\right)=(0.0616419: 0.474179: 0.464179)$.

$\operatorname{Var}\left(p_{1}\right)=0.00255598, \operatorname{Var}\left(p_{2}\right)=0.00602761, \operatorname{Var}\left(1-p_{1}-p_{2}\right)=0.0149735$

(ii) Typical Antipsychotic Drugs (TAD)

$$
\begin{aligned}
& \frac{1-p_{1}-p_{2}}{p_{1}}=12.186492 \quad, \quad \frac{1-p_{1}-p_{2}}{p_{2}}=0.635312 \\
& \frac{p_{1}}{p_{2}}=0.0521325
\end{aligned}
$$

Imposing the constraints

$\left(p_{1}: p_{2}: 1-p_{1}-p_{2}\right)=(0.0308943: 0.592612: 0.376494)$.

$\operatorname{Var}\left(p_{1}\right)=0.00157417, \operatorname{Var}\left(p_{2}\right)=0.00590434, \operatorname{Var}\left(1-p_{1}-p_{2}\right)=$ 0.0117654 . 
Note that the Kolmogorov Smirnov (KS) test between the initial frequencies in table 1 and the obtained density from equation (1) is $\mathrm{D}=0.66667$, p-value $=0.6$.

Next we substitute the values of $p_{1}, p_{2}, p_{3}$ for each of AAD and TAD into eq (2) and obtained the probability mass function. The graph of probability mass function against BMI is then plotted to reveal the induced behavioural growth in weight gain as a result of AAD and TAD intake.

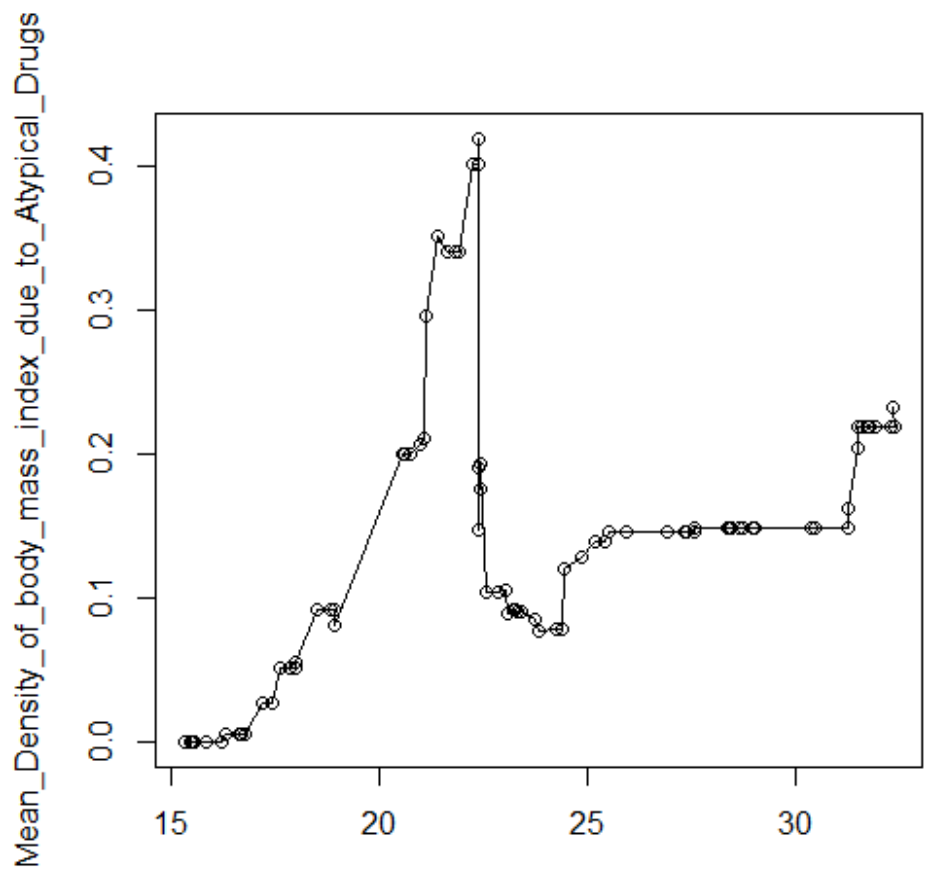

Body_mass_index

Figure 1. Mean Density of body weight gain induced by Atypical Antipsychotic Drugs

Figure 1, shows the growth of body weights among patient treated with AAD. The body weights continuously and steadily build up as the patient continues to take the drug. Almost $40 \%$ of the patients experiences increase in body weights that is within the normal range (BMI 18-24) though $10 \%$ of the patients still experiences underweight (BMI <18) despite taking the drug. We had lots of patients whose BMI are stabilized at 23, a few of the patients still experiences abnormal increase in body weights as the BMI increases beyond 24 . The percentage of patients who have experienced the increase in BMI beyond 25, continuously increases from $9 \%$ to almost $22 \%$. This increase however reveals a BMI beyond 30 implies that atypical can actually cause obesity and so it should be monitored during administration so as not to create other problems. 


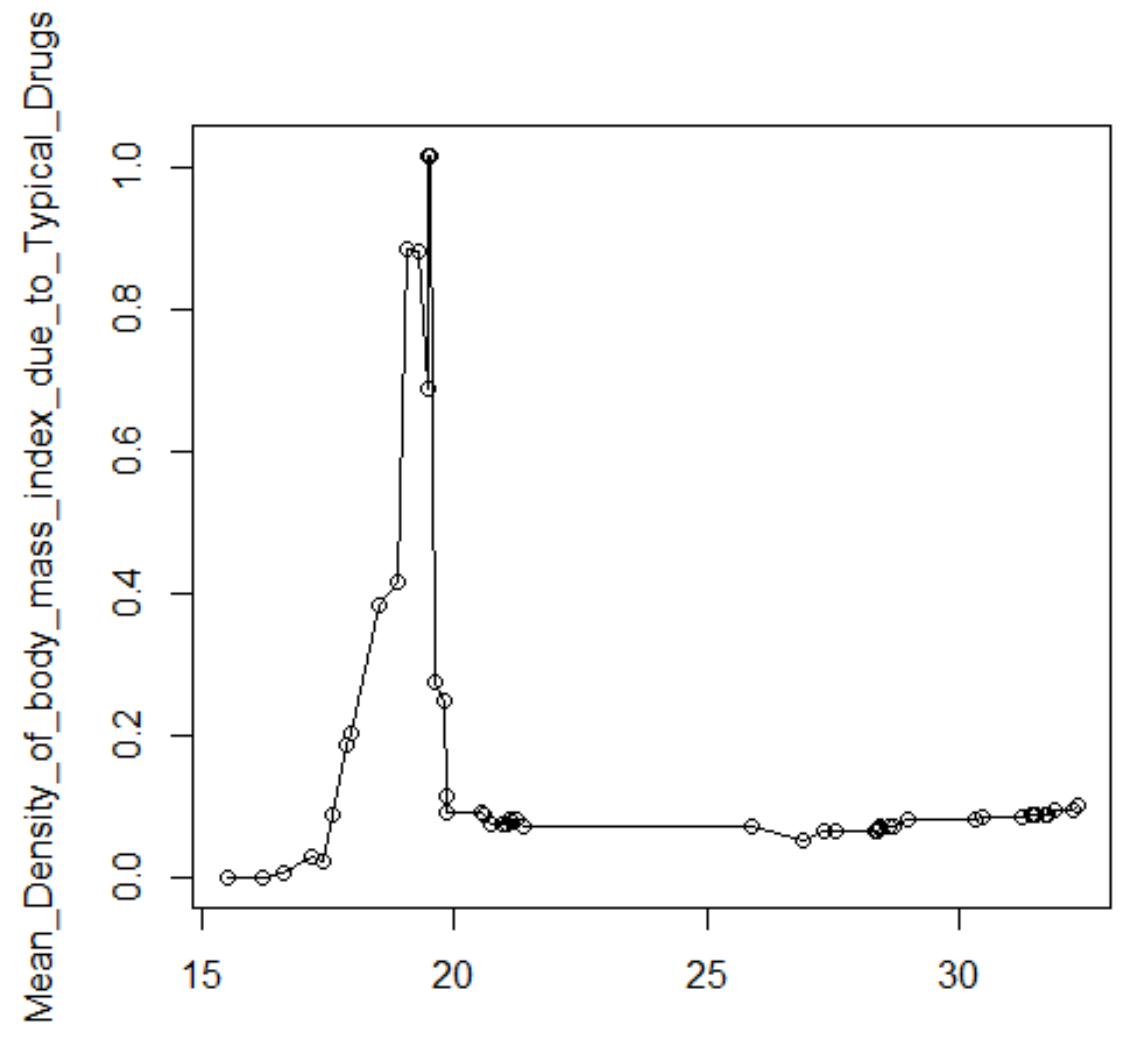

Body_mass_index

Figure 2. Mean Density of body weight gain induced by Typical Antipsychotic Drugs.

Similarly, from figure 2, almost all the patients using typical antipsychotic drugs experiences increase in body weights with less than $5 \%$ patients being underweight despite taking the drug. This increase was however still within the normal BMI range (18-24). The figure further shows that between $10 \%$ and $13 \%$ of the patients experiences overweight. 


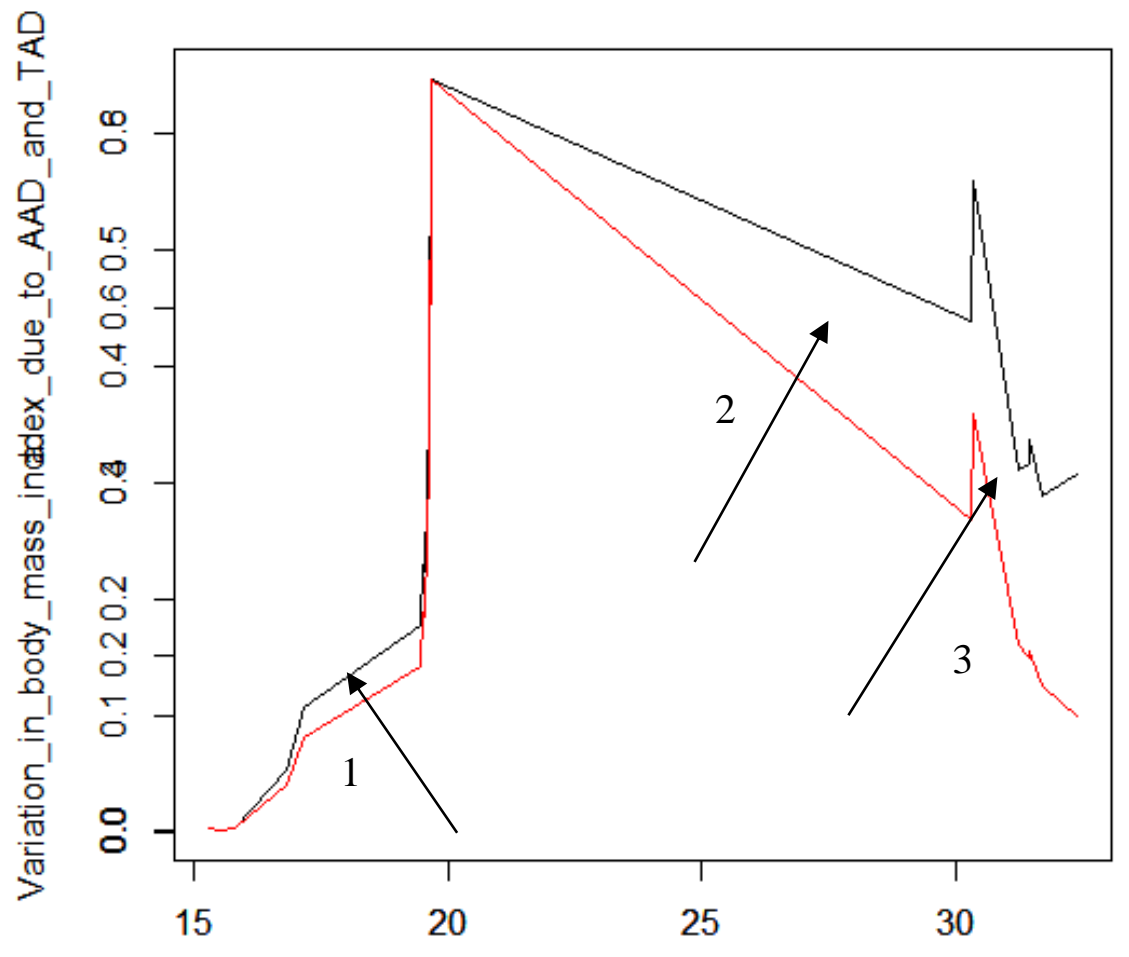

Body_mass_index

Figure 3. Variation in body weight gain due to Atypical Antipsychotic Drugs (black) and Typical Antipsychotic Drugs (red)

Comparing the variation in body weight gain as a result of the usage of atypical and typical antipsychotic drugs, figure 3 reveals that the density of body weight gain due to atypical drugs is slightly higher than that of the typical drugs (see arrow 1) within the BMI $15-20$ almost $20 \%$ of the patients given atypical and typical experienced this slight variation. However, there is no clear variation in body weight gain at BMI 20. Beyond BMI of 20, the variation in body weight gain between patients taking atypical and typical begins to show a gradual wide gap (see arrow 2) with atypical patients experiencing a noticeably higher body weight gain compared to their counterparts that are treated with typical drugs. About $40 \%$ $-80 \%$ of the patients experienced this wide body weight gain differential between patients with atypical and typical drugs. The variation gap was however reduced at BMI 30 and begins to vary again beyond BMI of 30 (see arrow 3). 


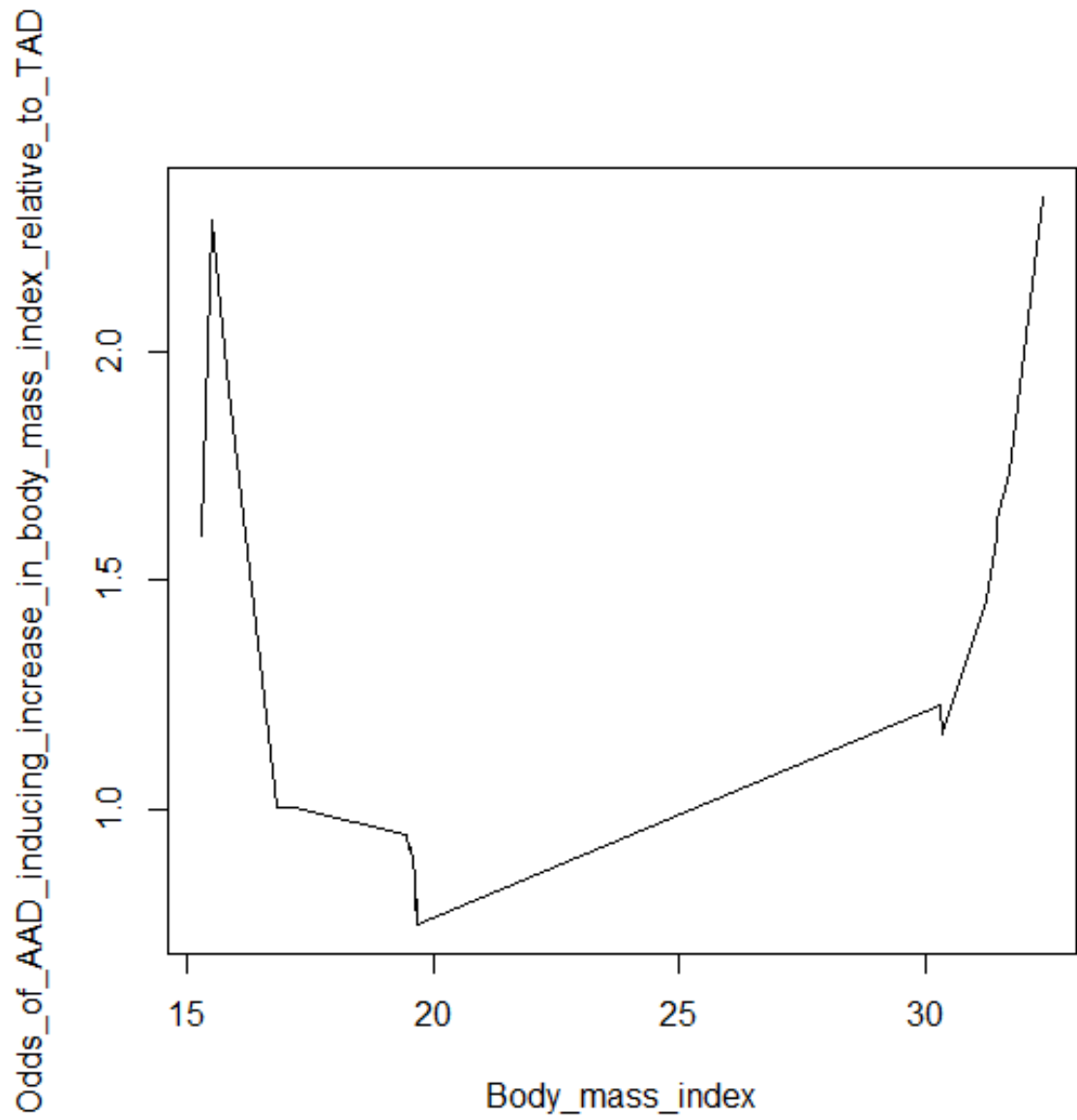

Figure 4. Odds of Atypical Antipsychotic Drugs inducing body weight gain relative to Typical Antipsychotic Drugs

The odd of AAD causing a significant change in patients' weight relative to TAD grows sharply from normal weight to obesity. Nutritional kits are however recommended for patients with AAD; though few patients with TAD can also be benefitted. Finally, investigating the proportion of patients with weight gain due to AAD relative to TAD per disease status, via the continuation ratio approach using the likelihood function (2), the following table was constructed.

Table 2: Proportion of weight gain due to AAD relative to TAD

\begin{tabular}{|c|l|}
\hline Disease Status & Proportion of weight gain \\
\hline Schizophrenia & $69.29 \%$ \\
\hline Bipolar & $50 \%$ \\
\hline Anxiety & $60 \%$ \\
\hline Overall & $61.47 \%$ \\
\hline
\end{tabular}




\section{Conclusion}

The study reveals that both TAD and AAD has the tendency of increasing BMI of the patients while patients with AAD showing higher induced body weight gain among the underweight and TAD showing same among patients of normal BMI. However, beyond the normal BMI range, the odds of AAD inducing a high abnormal increase in BMI and is more pronounced than that of the TAD. The odd of weight gain due to $\mathrm{AAD}$ relative to $\mathrm{TAD}$ via continuation ratio approach also confirms the graphical solution obtained. Thus, it is possible to recommend that nutritional control among psychiatric patients should be given thorough follow up beyond the hospital environment. This will prevent the psychiatric patients from developing cardiovascular related issues that can cause more problems beyond the current illness of the patients. Hence, the follow up of nutritional control should be taken into consideration: Mentally ill patients along with their relatives should be counseled to adopt healthy eating habits and encouraged to improve their diets in order to prevent other nutritional diseases. Reduction of weight for those that are obese can be achieved and maintained through healthy eating and increased physical activity. Also, there is a need for good nutrition and diversity in diets in terms of increased intake of foods rich in fibers, fruits and vegetables. Encouragement of small- and large-scale production of important edible fruits and vegetables locally to make the product available and cheap. Dieticians should be involved in management of these patients' foods and also to develop and implement weight management program to the patients.

\section{Acknowledgements}

We would like to appreciate the useful suggestions of the anonymous reviewers. Those suggestions have not only improved the paper, it has also enriched our understanding of the work.

\section{References}

1. Agboola A.A, Esan O.T. and Soyinka A. T. (2020). Work Productivity and Quality of life Mental Health Patients Attending Neuropsychiatric Hospital, Aro. Global Journal of Health Science, 12(4):81-93.

2. Allison D.B. and Pi-Sunyer F.X. (1995). Obesity treatment examining the premises. Endocrine Practice, 1: 353 -364.

3. Baptista T., Kin N., Beaulieu S. and De-Baptista E. (2002b). Obesity andrelated metabolic abnormalities during antipsychotic drug administration: mechanisms, management and research perspectives. Pharmacopsychiatry, 35:205-219. 
4. Baptista T., Zarate J., Joober R., Colasante C., Beaulieu S., Paez, X. and Hernandez L. (2004a). Drug induced weight gain, an impediment to successful pharmacotherapy: Focus on antipsychotics. Current Drug Targets, 5:279-299.

5. Bernstein J.G. (1987). Induction of obesity by psychotropic drugs. Ann NY Academy science, 499; 203- 215.

6. Chukwujekwu D.C. and Olose O.E. (2019). Body Mass Index Changes of Patients on Antipsychotics: A Comparison between Typical and Atypical Antipsychotics. Journal of Psychiatry and Psychiatric Disorders, 3(1): 6-13.

7. Green A., Patel J., Goisman R., Allison D., and Blackburn G (2000). Weight gain from novel antipsychotic drugs: need for action. General Hospital Psychiatry, 22:224-235.

8. Henderson D. (2002). Atypical antipsychotic-induced diabetes mellitus: How strong is the evidence? CNS Drugs, 16:77-89

9. Holt R., Peveler R., and Byrne C. (2004) Schizophrenia, the metabolicsyndrome and diabetes. Diabetes Medicine, 21:515-523

10. Johnson, R.A. and Wichern, D.W. (2006). Applied Multivariate Statistical Analysis. Englewood Cli_s, NJ: Prentice-Hall, Inc.

11. Lopez-Munoz, F., Tracy, D.K., Povedano-Montero, F.J., Breedvelt, J., Garcia-Pacious, J., Fernandez-Martin, M.P., Rubio, G., Alamo, C. (2019). Trends in the scientific literature on Atypical Antipsychotic Drugs in United Kingdom. Therapeutic Advances in Psychopharmacology, 9:1-12.

12. Mas-Expósito L., Mazo A., Emeterio M.S., Teixidó M., and LalucatJo L. (2012). Physical health and schizophrenia in clinical practice guidelines and consensus statements. Journal addiction Research and Therapy, S8 (001):1-5

13. Mood A. (1974). Introduction to the theory of statistics (McGraw-Hi11) series in probability and statistics). ISBN 0-07-042864-6.

14. Suhasini S.R (2010). Lecture Notes on Advanced Statistical Inference. suhasini.subbarao@stat.tamu.edu. 